\title{
Temperatura, baixo oxigênio e 1-metilciclopropeno na conservação da qualidade de caqui "Fuyu"
}

\author{
Temperature, low oxygen and 1-methylcyclepropene on the quality conservation of 'Fuyu' persimmon
}

\author{
Josuel Alfredo Vilela Pinto ${ }^{\mathrm{I}}$ Auri Brackmann ${ }^{\mathrm{II}}$ Cristiano André Steffens ${ }^{\mathrm{III}}$ Anderson Weber ${ }^{\mathrm{II}}$ \\ Ana Cristina Eisermann ${ }^{\text {II }}$
}

\section{RESUMO}

\begin{abstract}
O objetivo do presente trabalho foi o de avaliar o efeito de diferentes níveis de $\mathrm{O}_{2}, \mathrm{CO}_{2}$ e aplicação de 1-MCP (1metilciclopropeno) na temperatura de $10^{\circ} \mathrm{C}$ sobre a qualidade do caqui "Fuyu" armazenado em atmosfera controlada. Os tratamentos avaliados originaram-se da combinação de dois pontos de colheita (verde-amarelo e amarelo) e condições de armazenamento com: $20,8 \mathrm{kPa} \mathrm{O}_{2}+<0,5 \mathrm{kPa} \mathrm{CO}_{2}$ (Armazenamento Refrigerado - AR); 20,8kPa $\mathrm{O}_{2}+<0,5 \mathrm{kPa}$ $\mathrm{CO}_{2}$ mais aplicação de 1-MCP; 2,0kPa $\mathrm{O}_{2}+\mathrm{CO}_{2}$ livre; $1,0 \mathrm{kPa}$ $\mathrm{O}_{2}+\mathrm{CO}_{2}$ livre; $2,0 \mathrm{kPaO} \mathrm{O}_{2}+\mathrm{CO}_{2}$ livre mais aplicação de 1$\mathrm{MCP}$; >10,0 $\mathrm{kPaO} \mathrm{O}_{2}+12,0 \mathrm{kPaCO}$; >10,0kPa $\mathrm{O}_{2}+6,0 \mathrm{kPa}$ $\mathrm{CO}_{2}$, todos à temperatura de $10^{\circ} \mathrm{C}$; além de $20,8 \mathrm{kPa} \mathrm{O}_{2}+$ $<0,5 \mathrm{kPa} \mathrm{CO}_{2}$ (AR) $a-0,5^{\circ} \mathrm{C}$. Na saída da câmara, a menor incidência de escurecimento da casca foi observada nos frutos armazenados $a-0,5^{\circ} \mathrm{C}$. O armazenamento à temperatura de $10^{\circ} \mathrm{C}$, especialmente nas condições de $1,0 \mathrm{kPaO} \mathrm{O}_{2}+\mathrm{CO}_{2}$ livre e 2,0 $\mathrm{kPaO} \mathrm{O}_{2}+\mathrm{CO}_{2}$ livre mais aplicação de 1-MCP, proporcionou maior porcentagem de frutos firmes e menor incidência de podridão em caqui "Fuyu” após 17 dias de armazenamento.
\end{abstract}

Palavras-chave: pós-colheita, atmosfera controlada, etileno.

\section{ABSTRACT}

The present research was aimed at evaluating the effect of different levels of $\mathrm{O}_{2}, \mathrm{CO}_{2}$ and application of 1-MCP (1-methylcyclepropene) on the quality of 'Fuyu' persimmon stored in controlled atmosphere at $10^{\circ} \mathrm{C}$. The treatments evaluated came from the combination of two harvest stages (green-yellow and yellow) and storage conditions with: $20.8 \mathrm{kPaO} \mathrm{O}_{2}+<0.5 \mathrm{kPa} \mathrm{CO}$ (Cold Storage - CS); $20.8 \mathrm{kPaO}$ $+<0.5 \mathrm{kPaCO} \mathrm{CO}_{2}$ plus application of 1-MCP; $2.0 \mathrm{kPaO} \mathrm{O}_{2}+$ free $\mathrm{CO}_{2} ; 1.0 \mathrm{kPaO} \mathrm{O}_{2}+$ free $\mathrm{CO}_{2} ; 2.0 \mathrm{kPaO} \mathrm{O}_{2}+$ free $\mathrm{CO}_{2}$ plus application of $1-\mathrm{MCP} ;>10.0 \mathrm{kPa} \mathrm{O}_{2}+12.0 \mathrm{kPa} \mathrm{CO} \mathrm{C}_{2} ;>10.0 \mathrm{kPa}$ $\mathrm{O}_{2}+6.0 \mathrm{kPaC \textrm {CO } _ { 2 }}$, all treatment at $10^{\circ} \mathrm{C}$; and $20.8 \mathrm{kPa} \mathrm{O}_{2}+$ $<0.5 \mathrm{kPaCO} \mathrm{CO}_{2}(\mathrm{CS})$ at $-0.5^{\circ} \mathrm{C}$. At chamber opening, incidence of skin browning was lower on fruits stored at $-0.5^{\circ} \mathrm{C}$. Fruits stored at $10^{\circ} \mathrm{C}$, especially in the controlled atmosphere conditions $1.0 \mathrm{kPaO} \mathrm{O}_{2}+$ free $\mathrm{CO}_{2}$ and $2.0 \mathrm{kPaO}_{2}+$ free $\mathrm{CO}_{2}$ plus application of 1-MCP, showed higher percentage of firm fruits and lower decay incidence in 'Fuyu' persimmon after 17 days at of storage $10^{\circ} \mathrm{C}$.

Key words: postharvest, controlled atmosphere, ethylene.

\section{INTRODUÇÃO}

A produção brasileira de caqui está aumentando anualmente, em virtude da entrada em produção de novos pomares. São Paulo é o maior produtor brasileiro de caqui, sendo o Rio Grande do Sul o segundo colocado. De acordo com a EMATER/ RS, a produção gaúcha de caqui, em 2003, foi de 23.134 toneladas, produzidas em cerca de 1.020ha, destacandose a região da Serra Gaúcha como a maior produtora. Dentre as cultivares exploradas comercialmente, a "Fuyu" é uma das mais cultivadas, e a de maior importância no mercado internacional.

A colheita brasileira de caqui é realizada quando os países do hemisfério norte encontram-se no período de entressafra. Dessa forma, surge a possibilidade de os produtores exportarem parte da sua produção para esses países, especialmente para a

IUniversidade Federal de Santa Maria (UFSM), Santa Maria, RS, Brasil.

IIDepartamento de Fitotecnia, UFSM, 97105-900, Santa Maria, RS, Brasil. E-mail: brackman@ccr.ufsm.br. Autor para correspondência.

IIIUniversidade do Estado de Santa Catarina, Lages, SC, Brasil. 
Europa. No entanto, para isso ser possível, o transporte deve ser realizado em condições que possibilitem a manutenção da qualidade dos frutos. As tentativas de transporte marítimo em containeres refrigerados para a Europa, realizadas até o momento, não proporcionaram os resultados desejados, pois os frutos chegaram ao mercado europeu com elevada porcentagem de amolecimento e incidência de escurecimento da casca, principalmente após a exposição à temperatura ambiente por um ou dois dias. $\mathrm{O}$ amolecimento rápido dos frutos após a exposição à temperatura ambiente foi constatado por DONAZZOLO \& BRACKMANN (2002). O amolecimento é a mais proeminente mudança deteriorativa em caquis e está diretamente ligada ao amadurecimento e ocorre devido à degradação dos constituintes da parede celular, como as pectinas e hemiceluloses (DA SILVA et al., 1997). Já o escurecimento da casca é causado pela atividade da enzima polifenol oxidase, sendo influenciada por vários fatores de pré-colheita e de pós-colheita (PARK, 1997). Já LEE et al. (1999) observaram que o escurecimento da epiderme ocorreu principalmente nas cicatrizações ou junto a rachaduras e fendas da epiderme.

A baixa temperatura, se mal usada, pode provocar injúrias causadas pelo frio (SARGENT et al., 1993). Estes autores verificaram que a curva de incidência de dano pelo frio descreve-se como uma parábola, sendo que a maior incidência ocorre na temperatura de $5^{\circ} \mathrm{C}$. Dessa forma, para conservação por curtos períodos, a temperatura de $20^{\circ} \mathrm{C}$ é a mais recomendada; no entanto, esta acelera a maturação. Para uma conservação mais prolongada, os autores indicam uma temperatura situada em torno de $0^{\circ} \mathrm{C}$, pois, nesta condição de armazenamento, não se detectou dano pelo frio. CRISOSTO et al.(2004) também encontraram maior suscetibilidade de dano pelo frio em caqui "Fuyu" numa faixa de temperatura entre $5 \mathrm{e}$ $15^{\circ} \mathrm{C}$. No entanto, WOOLF et al. (1997), armazenando caqui "Fuyu" em temperatura de $0^{\circ} \mathrm{C}$ por 6 meses, também observaram danos ocasionados pelo frio. $\mathrm{O}$ rápido resfriamento a temperaturas baixas $\left(-1^{\circ} \mathrm{C}\right)$ pode ser uma das causas da incidência de escurecimento da polpa (LEE, 2001). Porém, a exposição dos frutos a altas temperaturas, durante a fase de maturação, resulta numa rápida perda de firmeza de polpa (HARIMA et al., 2003).

O período de conservação do caqui no sistema chamado armazenamento refrigerado, em que são controladas apenas a temperatura e umidade relativa, é muito curto. Para prolongar o armazenamento, pode ser utilizada a atmosfera controlada. A alteração da atmosfera do ambiente de armazenamento visa a contribuir com a baixa temperatura para reduzir ainda mais o metabolismo dos frutos. Altas concentrações de $\mathrm{CO}_{2}$ podem inibir a síntese e ação do etileno (DONG et al., 1992) e baixas concentrações de $\mathrm{O}_{2}$ interrompem a transformação de ACC em etileno, pois a enzima ACCoxidase utiliza $\mathrm{O}_{2}$ (TAIZ \& ZAIGER, 1998), apresentando também efeito direto em diversas outras enzimas envolvidas no amadurecimento dos frutos (BRACKMANN \& CHITARRA, 1998), ainda reduzindo a ocorrência de podridão (PRUSKY et al., 1997).

Não se dispõe de informações precisas a respeito de concentração de gases ideais para o armazenamento de caqui "Fuyu", principalmente em temperaturas elevadas. No entanto, BRACKMANN et al. (1997) armazenaram caqui "Fuyu" por três meses nas condições de $16 \mathrm{kPa}$ de $\mathrm{O}_{2}+15 \mathrm{kPa}$ de $\mathrm{CO}_{2}$, mantendo baixos níveis de escurecimento e boa firmeza de polpa na temperatura de $0,5^{\circ} \mathrm{C}$. A condição $2 \mathrm{kPa}$ de $\mathrm{O}_{2}+8 \mathrm{kPa}$ de $\mathrm{CO}_{2}$ foi a mais adequada, conforme BRACKMANN \& SAQUET (1995), para as cultivares “Taubaté", "Bauru" e "Fuyu". A incidência de escurecimento da epiderme foi reduzida quando LEE et al. (1993) usaram a condição de $2 \mathrm{kPa}$ de $\mathrm{O}_{2}+5 \mathrm{kPa}$ de $\mathrm{CO}_{2}$. VIDRIH et al. (1990) obtiveram frutos com maior firmeza de polpa com $2 \mathrm{kPa}$ de $\mathrm{O}_{2}+3 \mathrm{kPa}$ de $\mathrm{CO}_{2}$, mantendo a qualidade por até três meses. GORINI \& TESTONI (1988) recomendam para variedades nãoadstringentes condições entre 2 a $3 \mathrm{kPa}$ de $\mathrm{O}_{2}+5$ e $8 \mathrm{kPa}$ de $\mathrm{CO}_{2}$. Estes mesmos autores citam o uso de alto $\mathrm{CO}_{2}(12 \mathrm{kPa})$ para retardar o amolecimento do caqui; no entanto, ressaltam a possibilidade da ocorrência de escurecimento interno e de sabor alcoólico nos frutos.

$\mathrm{O}$ etileno interfere na manutenção da qualidade do caqui durante o armazenamento, devido a este ser um fruto climatérico, em que a senescência é fortemente induzida quando é armazenado na presença deste gás. O caqui é muito sensível à ação do etileno, respondendo à concentração de $1 \mathrm{ppm}$ (CRISOSTO et al., 2004), apesar de sua baixa produção $\left(<0,1 \mu 1 \mathrm{~kg}^{-1} \mathrm{~h}^{-1} \mathrm{a}\right.$ $0^{\circ} \mathrm{C} \mathrm{e} 0,1-0,5 \mu 1 \mathrm{~kg}^{-1} \mathrm{~h}^{-1}$ a $\left.20^{\circ} \mathrm{C}\right)$. O etileno pode induzir a perda de firmeza de polpa, a diminuição de pectina total e a mudança na coloração da epiderme. Para reduzir esses problemas ocasionados pelo etileno, podem ser utilizados absorvedores deste fitohormônio, bem como 1-metilciclopropeno (1-MCP).

Ciência Rural, v.37, n.5, set-out, 2007. 
O composto 1-metilciclopropeno (1-MCP) liga-se ao receptor do etileno (SISLER \& SEREK, 1999), inibindo a ação da presença desse fitohormônio (MULLINS et al., 2000) e, conseqüentemente, as respostas dos frutos (BLANKENSHIP, 2001). A ligação do 1-MCP às moléculas receptoras do etileno elimina, como propõe MULLINS et al. (2000), a regulação precisa da rota de síntese desse fitohormônio.

Diante disso, o presente trabalho teve como objetivo avaliar o efeito de diferentes condições de armazenamento em atmosfera controlada e de aplicação de 1-MCP (1-metilciclopropeno) sobre a qualidade do caqui "Fuyu", armazenado na temperatura de $10^{\circ} \mathrm{C}$.

\section{MATERIAL E MÉTODOS}

O experimento foi conduzido no Núcleo de Pesquisa em Pós-Colheita (NPP) do Departamento de Fitotecnia da Universidade Federal de Santa Maria (UFSM). Os frutos utilizados na condução do experimento foram colhidos em um pomar comercial de Caxias do Sul, RS. O delineamento experimental utilizado foi o inteiramente casualizado, com três repetições, sendo as unidades experimentais compostas por 20 frutos e os tratamentos arranjados em esquema bifatorial.

Os tratamentos avaliados originaram-se da combinação de dois pontos de colheita (verde-amarelo e amarelo) e condições de armazenamento com: $20,8 \mathrm{kPa}$ $\mathrm{O}+<0,5 \mathrm{kPaCO}$ (Armazenamento Refrigerado - AR); $20^{2}, 8 \mathrm{kPa} \mathrm{O}_{2}+<0,5 \mathrm{kPa} \mathrm{CO}_{2}$ mais aplicação de $1 \mu \mathrm{L} \mathrm{L}^{-1} \mathrm{de}$ 1-MCP; 2,0 $\mathrm{kPaO}_{2}+\mathrm{CO}_{2}$ livre; $1,0 \mathrm{kPaO}_{2}+\mathrm{CO}_{2}$ livre; 2,0 $\mathrm{kPaO}_{2}+\mathrm{CO}_{2}$ livre mais aplicação de $1 \mu \mathrm{L} \mathrm{L}^{-1}$ de 1-MCP; $>10,0 \mathrm{kPaO}_{2}+12,0 \mathrm{kPaCO}_{2} ;>10,0 \mathrm{kPaO}_{2}+6,0 \mathrm{kPaCO}_{2}$, todos à temperatura de $10^{\circ} \mathrm{C}$; além de $20,8 \mathrm{kPa} \mathrm{O}_{2}+$ $<0,5 \mathrm{kPa} \mathrm{CO}$ (AR) a $-0,5^{\circ} \mathrm{C}$. A expressão $\mathrm{CO}_{2}$ livre significa que a concentração deste gás não foi controlada, sendo portanto estabelecida pela respiração dos frutos. O 1-MCP foi aplicado em uma minicâmara experimental, hermeticamente fechada, durante 24 horas, na temperatura de armazenamento. Os teores de $\mathrm{CO}_{2}$, preestabelecidos nos tratamentos, foram obtidos mediante injeção deste gás nas minicâmaras, provenientes de cilindros de alta pressão. O processo respiratório dos frutos resultou no consumo de $\mathrm{O}_{2} \mathrm{e}$ no aumento de $\mathrm{CO}_{2} \mathrm{e}$, para a manutenção dos níveis estabelecidos, foram realizadas diariamente análises e correções dos gases. A medição e correção das pressões parciais de $\mathrm{O}_{2}$ e $\mathrm{CO}_{2}$ foram efetuadas automaticamente por um equipamento de análise e controle de gases da marca Kronenberger/Climasul. O $\mathrm{O}_{2}$ consumido pela respiração dos frutos foi compensado pela injeção de ar nas minicâmaras. $\mathrm{OCO}_{2}$, quando em excesso, foi eliminado circulando-se o gás das minicâmaras por uma solução de hidróxido de potássio a $40 \%$. As temperaturas das câmaras foram reguladas por meio de termostatos eletrônicos e acompanhadas diariamente através de termômetros com bulbo de mercúrio inseridos na polpa de um fruto.

Os frutos permaneceram armazenados por 17 dias, simulando o período médio necessário para o transporte marítimo dos frutos até a Europa. As avaliações de consistência da polpa, índice de escurecimento e podridão foram realizadas na saída da câmara, ao terceiro e ao sexto dias de exposição a $20^{\circ} \mathrm{C}$. A respiração e a produção de etileno foram avaliadas do primeiro até o quinto dia de exposição a $20^{\circ} \mathrm{C}$. Foram utilizadas as seguintes metodologias: consistência da polpa, através de um teste subjetivo aplicando-se uma leve pressão sobre os frutos com os dedos, sendo considerados moles os que apresentavam área amolecida superior a $1 \mathrm{~cm}$ de diâmetro, atribuindo porcentagem à área amolecida para cada fruto; escurecimento da casca, determinada através dos seguintes índices: $0=$ sem presença de escurecimento da casca; 1 = até $10 \%$ da superfície da casca escurecida; $2=>10$ a $20 \%$ da superfície da casca do fruto escurecida; $3=>20$ a $30 \%$ da superfície escurecida; e $4=>30 \%$ da superfície da casca escurecida. $O$ índice médio foi calculado através da soma dos produtos do número de frutos pelos seus respectivos níveis de escurecimento, dividido pelo número total de frutos da amostra. A incidência de podridões, foi avaliada através da contagem dos frutos que apresentavam lesões superiores a $5 \mathrm{~mm}$ de diâmetro com características de ataque por fungos. Para a determinação da respiração, os frutos foram colocados em vidros fechados hermeticamente com capacidade de cinco litros, sendo, após duas horas, aproximadamente, determinada a concentração de $\mathrm{CO}_{2}$ através de um analisador de fluxo contínuo de gases, marca Agri-Datalog. Para a determinação da produção de etileno, utilizou-se um cromatógrafo a gás, equipado com coluna poropak $\mathrm{Ne}$ detector de ionização de chama, com uma temperatura da coluna, injetor e detector de 90,120 e $200^{\circ} \mathrm{C}$, respectivamente. Para o cálculo da respiração e produção de etileno, levou-se em consideração, além da concentração de $\mathrm{CO}_{2} \mathrm{e} \mathrm{C}_{2} \mathrm{H}_{4}$, o volume do recipiente, a massa de frutos e o tempo em que os recipientes permaneceram fechados.

Os dados expressos em porcentagem foram transformados pela fórmula $\operatorname{arc}$.sen $\sqrt{x / 100}$ antes 
de serem submetidos à análise da variância. As médias foram comparadas entre si pelo teste de Duncan em nível de 5\% de probabilidade de erro.

\section{RESULTADOS E DISCUSSÃO}

Na saída dos frutos das câmaras, verificouse que o armazenamento a $-0,5^{\circ} \mathrm{C}$ e em atmosfera controlada proporcionou maior porcentagem de frutos firmes (Tabela 1). Resultados semelhantes foram obtidos por BRACKMANN \& SAQUET (1995). O armazenamento nas condições de $>10 \mathrm{kPaO}_{2}+6,0 \mathrm{kPa}$ $\mathrm{CO}_{2}, 1,0 \mathrm{kPaO}_{2}+\mathrm{CO}_{2}$ livre e $2,0 \mathrm{kPa} \mathrm{O}_{2}+\mathrm{CO}_{2}$ livre mais aplicação de 1-MCP permitiu a manutenção da consistência da polpa dos frutos, não diferindo estatisticamente da condição de armazenamento $-0,5^{\circ} \mathrm{C}$ (Tabela 1). O efeito positivo da aplicação de 1-MCP, na manutenção da firmeza da polpa de caquis "Triumph", foi relatado por BEN-ARIE et al. (2001). Após três dias de exposição dos frutos a $20^{\circ} \mathrm{C}$, os frutos armazenados $\mathrm{a}-0,5^{\circ} \mathrm{C}$ apresentaram rápida perda da consistência da polpa (Tabela 1). Esses resultados sugerem que o armazenamento em baixa temperatura pode desencadear o processo de amadurecimento ou provocar algum dano fisiológico aos frutos, reduzindo a firmeza da polpa drasticamente após a exposição à temperatura ambiente. No entanto, o armazenamento em condições de atmosfera controlada a $10^{\circ} \mathrm{C}$ permitiu a manutenção de mais de $70 \%$ dos frutos com consistência firme após a exposição por três dias à temperatura de $20^{\circ} \mathrm{C}$, não havendo diferença estatística entre as condições de atmosfera controlada. Mesmo após seis dias a $20^{\circ} \mathrm{C}$, observou-se mais de $60 \%$ de frutos firmes no tratamento com $2,0 \mathrm{kPa} \mathrm{O}_{2}+\mathrm{CO}_{2}$ livre mais aplicação de 1-MCP, não diferindo estatisticamente da condição de $1,0 \mathrm{kPa} \mathrm{O}_{2}+\mathrm{CO}_{2}$ livre e $2,0 \mathrm{kPa} \mathrm{O}_{2}+\mathrm{CO}_{2}$ livre (Tabela 1). Menor incidência de escurecimento da casca foi observada nos frutos armazenados a $-0,5^{\circ} \mathrm{C}$ e em $1,0 \mathrm{kPa} \mathrm{O}_{2}+\mathrm{CO}_{2}$ livre, na saída da câmara (Tabela 2). Entretanto, com a exposição dos frutos a $20^{\circ} \mathrm{C}$ a incidência de escurecimento aumentou significativamente nos frutos armazenados em atmosfera controlada e a $-0,5^{\circ} \mathrm{C}$. Os frutos armazenados a $10^{\circ} \mathrm{C}$ em $1,0 \mathrm{kPa} \mathrm{O}_{2}+\mathrm{CO}_{2}$ livre apresentaram os menores valores de escurecimento durante o período analisado de exposição a $20^{\circ} \mathrm{C}$ (Tabela 2). Esses resultados discordam de LEE et al. (2003), que verificaram maior incidência de escurecimento da casca de caquis "Fuyu" com a utilização de pressões parciais baixas de $\mathrm{O}_{2}$.

De acordo com os resultados, a colheita no ponto verde-amarelo proporcionou maior porcentagem de frutos firmes e menores índices de escurecimento da casca (Tabelas 1 e 2). A ocorrência de frutos podres não diferiu entre os tratamentos avaliados, permanecendo inferior a $5 \%$ na saída da câmara e após três dias de exposição a $20^{\circ} \mathrm{C}$ (Tabela 3). Já após 6 dias de exposição a $20^{\circ} \mathrm{C}$, os frutos submetidos a $2 \mathrm{kPa}$ de

Tabela 1 - Porcentagem de caqui cv. "Fuyu" com consistência firme após 17 dias de armazenamento e após a exposição a $20^{\circ} \mathrm{C}$. Santa Maria, RS, 2005.

\begin{tabular}{|c|c|c|c|c|c|c|c|c|c|c|}
\hline \multicolumn{2}{|c|}{ Condições de armazenamento } & \multicolumn{9}{|c|}{ Consistência firme (\%) } \\
\hline \multirow{2}{*}{$\begin{array}{c}\text { Temperatura } \\
{ }^{\circ} \mathrm{C}\end{array}$} & \multirow[b]{2}{*}{$\mathrm{O}_{2}+\mathrm{CO}_{2}(\mathrm{kPa})$} & \multicolumn{3}{|c|}{ Saída da câmara } & \multicolumn{3}{|c|}{3 dias a $20^{\circ} \mathrm{C}$} & \multicolumn{3}{|c|}{6 dias a $20^{\circ} \mathrm{C}$} \\
\hline & & $\begin{array}{c}\text { Verde } \\
\text { amarelo }\end{array}$ & Amarelo & Médias & $\begin{array}{l}\text { Verde- } \\
\text { amarelo }\end{array}$ & Amarelo & Médias & $\begin{array}{c}\text { Verde } \\
\text { amarelo }\end{array}$ & Amarelo & Médias \\
\hline 10 & $\mathrm{AR}^{*}$ & 0,0 & 0,0 & $0,0 \mathrm{~d}^{* * *}$ & 0,0 & 0,0 & $0,0 \mathrm{~d}$ & 0,0 & 0,0 & $0,0 \mathrm{~d}$ \\
\hline 10 & $\mathrm{AR}^{* *}$ & 62,2 & 28,5 & $45,40 \mathrm{c}$ & 22,7 & 9,52 & $16,11 \mathrm{c}$ & 20,32 & 4,76 & $12,54 \mathrm{bc}$ \\
\hline 10 & $2,0+$ livre & 100,0 & 71,4 & $85,71 b$ & 91,1 & 65,0 & $78,09 \mathrm{a}$ & 64,44 & 46,0 & $55,24 \mathrm{ab}$ \\
\hline 10 & $2,0+$ livre $* *$ & 93,3 & 90,4 & $91,90 \mathrm{ab}$ & 88,8 & 57,1 & $73,02 \mathrm{a}$ & 82,06 & 47,6 & $64,84 \mathrm{a}$ \\
\hline 10 & $>10+12$ & 97,7 & 76,1 & $86,98 \mathrm{~b}$ & 91,1 & 64,2 & $77,69 \mathrm{a}$ & 42,22 & 33,3 & $37,78 b$ \\
\hline 10 & $1,0+$ livre & 97,7 & 90,4 & $94,12 \mathrm{ab}$ & 90,0 & 71,4 & $80,71 \mathrm{a}$ & 52,78 & 38,1 & $45,44 \mathrm{ab}$ \\
\hline 10 & $>10+6,0$ & 93,3 & 95,2 & $94,28 \mathrm{ab}$ & 84,2 & 70,6 & $77,46 a$ & 57,30 & 15,0 & $36,19 b$ \\
\hline$-0,5$ & AR & 100,0 & 95,2 & $97,62 \mathrm{a}$ & 57,7 & 30,9 & $44,37 b$ & 22,22 & 0,0 & $11,11 \mathrm{c}$ \\
\hline Média & & $80,55 \mathrm{~A}$ & $68,45 \mathrm{~B}$ & & $65,73 \mathrm{~A}$ & $46,13 \mathrm{~B}$ & & $42,67 \mathrm{~A}$ & $23,12 \mathrm{~B}$ & \\
\hline $\mathrm{CV}$ & & & 13,87 & & & 22,39 & & & 40,43 & \\
\hline
\end{tabular}

*Armazenamento refrigerado; ** Aplicação de $1 \mu \mathrm{L} \mathrm{L}^{-1}$ de 1 -MCP durante 24 horas; *** Médias seguidas de mesma letra, minúscula na vertical e maiúscula na horizontal, não diferem entre si pelo teste de Duncan a $5 \%$. 
Tabela 2 - Índice de escurecimento da casca em caquis cv. "Fuyu" após 17 dias de armazenamento e durante a exposição a $20^{\circ} \mathrm{C}$. Santa Maria, 2005.

\begin{tabular}{|c|c|c|c|c|c|c|c|c|c|c|}
\hline \multicolumn{5}{|c|}{ Condições de armazenamento } & \multicolumn{6}{|c|}{ ndice de escurecimento $(1-4) * * * *$} \\
\hline \multirow{2}{*}{$\begin{array}{c}\text { Temperatura } \\
{ }^{\circ} \mathrm{C}\end{array}$} & \multirow[b]{2}{*}{$\mathrm{O}_{2}+\mathrm{CO}_{2}(\mathrm{kPa})$} & \multicolumn{3}{|c|}{ Saída da câmara } & \multicolumn{3}{|c|}{3 dias a $20^{\circ} \mathrm{C}$} & \multicolumn{3}{|c|}{6 dias a $20^{\circ} \mathrm{C}$} \\
\hline & & $\begin{array}{l}\text { Verde- } \\
\text { amarelo }\end{array}$ & Amarelo & Médias & $\begin{array}{l}\text { Verde- } \\
\text { amarelo }\end{array}$ & Amarelo & Médias & $\begin{array}{l}\text { Verde- } \\
\text { amarelo }\end{array}$ & Amarelo & Médias \\
\hline 10 & $\mathrm{AR} *$ & 0,09 & 0,52 & $0,31 a b^{* * *}$ & 0,75 & 0,94 & $0,84 b c$ & 1,00 & 1,11 & $1,06 \mathrm{~b}$ \\
\hline 10 & $\mathrm{AR}^{* *}$ & 0,31 & 0,38 & $0,34 \mathrm{a}$ & 1,03 & 1,05 & $1,04 \mathrm{~b}$ & 1,09 & 1,14 & $1,12 b$ \\
\hline 10 & $2,0+$ livre & 0,11 & 0,19 & $0,15 \mathrm{bcd}$ & 0,53 & 1,04 & $0,78 b c$ & 0,58 & 1,14 & $0,86 \mathrm{bc}$ \\
\hline 10 & $2,0+$ livre $^{* *}$ & 0,27 & 0,43 & $0,35 \mathrm{a}$ & 0,73 & 1,24 & $0,99 b$ & 0,80 & 1,43 & $1,11 \mathrm{~b}$ \\
\hline 10 & $>10+12$ & 0,11 & 0,33 & $0,22 \mathrm{abc}$ & 0,71 & 0,95 & $0,83 b c$ & 0,73 & 1,01 & $0,87 \mathrm{bc}$ \\
\hline 10 & $1,0+$ livre & 0,00 & 0,14 & $0,07 \mathrm{~cd}$ & 0,49 & 0,81 & $0,65 \mathrm{c}$ & 0,56 & 0,86 & $0,71 \mathrm{c}$ \\
\hline 10 & $>10+6,0$ & 0,20 & 0,30 & $0,25 \mathrm{ab}$ & 0,68 & 0,96 & $0,82 \mathrm{bc}$ & 0,81 & 1,06 & $0,93 b c$ \\
\hline$-0,5$ & AR & 0,02 & 0,00 & $0,01 \mathrm{~d}$ & 1,18 & 1,68 & $1,43 \mathrm{a}$ & 1,24 & 1,68 & $1,46 \mathrm{a}$ \\
\hline Média & & $0,14 \mathrm{~B}$ & $0,29 \mathrm{~A}$ & & $0,76 \mathrm{~B}$ & $1,08 \mathrm{~A}$ & & $0,85 \mathrm{~B}$ & $1,17 \mathrm{~A}$ & \\
\hline $\mathrm{CV}$ & & & 63,21 & & & & 24,49 & & 19 & \\
\hline
\end{tabular}

*Armazenamento refrigerado; ** Aplicação de $1 \mu \mathrm{L} \mathrm{L}^{-1}$ de 1-MCP durante 24 horas; *** Médias seguidas de mesma letra, minúscula na vertical e maiúscula na horizontal, não diferem entre si pelo teste de Duncan a $5 \%$; $* * * 1=$ até $10 \%$ da superfície da casca escurecida; $2=$ $>10$ a $20 \% ; 3=>20$ a $30 \%$; e $4=>30 \%$ da superfície da casca escurecida.

$\mathrm{O}_{2}+\mathrm{CO}_{2}$ livre apresentaram menor incidência de podridão (Tabela 3 ). Esses resultados concordam com NEUWALD (2004), que atribuía o baixo $\mathrm{O}_{2}$ como efeito fungistático, por inibir a germinação de esporos e o desenvolvimento dos fungos durante o período de armazenamento.

A produção de etileno e a respiração foram crescentes até o quarto dia de exposição a $20^{\circ} \mathrm{C}$, sendo que a interação entre ponto de maturação e a condição de armazenamento ocorreu até essa data na produção de etileno (Tabela 4). Já para a respiração, ocorreu interação apenas no quarto dia (Tabela 5). Nenhuma condição de armazenamento retardou o pico de produção de etileno e da respiração; no entanto, os frutos submetidos ao armazenamento refrigerado na temperatura de $-0,5^{\circ} \mathrm{C}$ tiveram a maior produção de

Tabela 3 - Porcentagem de caqui cv. "Fuyu" com podridão após 17 dias de armazenamento e após a exposição a $20^{\circ}$ C. Santa Maria, RS, 2005 .

\begin{tabular}{|c|c|c|c|c|c|c|c|c|c|c|}
\hline \multicolumn{2}{|c|}{ Condições de armazenamento } & \multicolumn{9}{|c|}{ Podridão (\%) } \\
\hline \multirow[b]{2}{*}{ Temperatura ${ }^{\circ} \mathrm{C}$} & \multirow[b]{2}{*}{$\mathrm{O}_{2}+\mathrm{CO}_{2}(\mathrm{kPa})$} & \multicolumn{3}{|c|}{ Saída da câmara } & \multicolumn{3}{|c|}{3 dias a $20^{\circ} \mathrm{C}$} & \multicolumn{3}{|c|}{6 dias a $20^{\circ} \mathrm{C}$} \\
\hline & & $\begin{array}{l}\text { Verde- } \\
\text { amarelo }\end{array}$ & Amarelo & Médias & $\begin{array}{l}\text { Verde- } \\
\text { amarelo }\end{array}$ & Amarelo & Médias & $\begin{array}{l}\text { Verde- } \\
\text { amarelo }\end{array}$ & Amarelo & Médias \\
\hline 10 & $\mathrm{AR}^{*}$ & 0,0 & 0,0 & $0,0 \mathrm{a}^{* * *}$ & 0,0 & 0,0 & $0,0 \mathrm{a}$ & 18,94 & 22,22 & $20,58 \mathrm{a}$ \\
\hline 10 & $\mathrm{AR}^{* *}$ & 0,0 & 0,0 & $0,0 \mathrm{a}$ & 0,0 & 0,0 & $0,0 \mathrm{a}$ & 9,05 & 19,05 & $14,05 \mathrm{ab}$ \\
\hline 10 & $2,0+$ livre & 0,0 & 0,0 & $0,0 \mathrm{a}$ & 0,0 & 0,0 & $0,0 \mathrm{a}$ & 0,00 & 4,76 & $2,38 \mathrm{c}$ \\
\hline 10 & $2,0+$ livre $^{* *}$ & 0,0 & 0,0 & $0,0 \mathrm{a}$ & 0,0 & 4,76 & $2,38 \mathrm{a}$ & 2,22 & 9,52 & $5,87 \mathrm{abc}$ \\
\hline 10 & $>10+12$ & 0,0 & 0,0 & $0,0 \mathrm{a}$ & 0,0 & 5,56 & $2,78 \mathrm{a}$ & 4,44 & 10,32 & $7,38 \mathrm{abc}$ \\
\hline 10 & $1,0+$ livre & 0,0 & 0,0 & $0,0 \mathrm{a}$ & 0,0 & 4,76 & $2,38 \mathrm{a}$ & 5,00 & 14,29 & $9,64 a b c$ \\
\hline 10 & $>10+6,0$ & 0,0 & 0,0 & $0,0 \mathrm{a}$ & 2,38 & 0,0 & $1,19 \mathrm{a}$ & 4,60 & 4,76 & $4,68 b c$ \\
\hline$-0,5$ & $\mathrm{AR}$ & 0,0 & 0,0 & $0,0 \mathrm{a}$ & 0,0 & 9,52 & $4,76 \mathrm{a}$ & 4,44 & 8,93 & $6,68 \mathrm{abc}$ \\
\hline Média & & $0,0 \mathrm{~A}$ & $0,0 \mathrm{~A}$ & & $0,77 \mathrm{~A}$ & $3,49 \mathrm{~A}$ & & $6,09 \mathrm{~A}$ & $11,73 \mathrm{~A}$ & \\
\hline $\mathrm{CV}$ & & & 0,0 & & & 82,65 & & & 86,95 & \\
\hline
\end{tabular}

*Armazenamento refrigerado; ** Aplicação de $1 \mu \mathrm{L} \mathrm{L}^{-1}$ de 1-MCP durante 24 horas; *** Médias seguidas de mesma letra, minúscula na vertical e maiúscula na horizontal, não diferem entre si pelo teste de Duncan a $5 \%$.

Ciência Rural, v.37, n.5, set-out, 2007. 
Tabela 4 - Produção de etileno em caquis cv. "Fuyu” após 17 dias de armazenamento e durante a exposição a $20^{\circ} \mathrm{C}$. Santa Maria, RS, 2005.

\begin{tabular}{|c|c|c|c|c|c|c|c|c|c|c|}
\hline \multirow{2}{*}{\multicolumn{2}{|c|}{ Condições de armazenamento }} & \multicolumn{9}{|c|}{ Produção de etileno $\left(\mu \mathrm{LC}_{2} \mathrm{H}_{4} \mathrm{~kg}^{-1} \mathrm{~h}^{-1}\right)$} \\
\hline & & \multicolumn{3}{|c|}{1 dia a $20^{\circ} \mathrm{C}$} & \multicolumn{3}{|c|}{2 dias a $20^{\circ} \mathrm{C}$} & \multicolumn{3}{|c|}{3 dias a $20^{\circ} \mathrm{C}$} \\
\hline $\begin{array}{c}\text { Temperatura } \\
{ }^{\circ} \mathrm{C}\end{array}$ & $\mathrm{O}_{2}+\mathrm{CO}_{2}(\mathrm{kPa})$ & $\begin{array}{l}\text { Verde- } \\
\text { amarelo }\end{array}$ & Amarelo & Médias & $\begin{array}{l}\text { Verde- } \\
\text { amarelo }\end{array}$ & Amarelo & Médias & $\begin{array}{l}\text { Verde- } \\
\text { amarelo }\end{array}$ & Amarelo & Médias \\
\hline 10 & $\mathrm{AR}^{*}$ & $0,17 \mathrm{Abc} * * *$ & $0,24 \mathrm{Ab}$ & 0,20 & 0,18 Acd & $0,28 \mathrm{Aab}$ & 0,23 & $0,23 \mathrm{Bb}$ & $0,44 \mathrm{Aa}$ & 0,33 \\
\hline 10 & $\mathrm{AR}^{* *}$ & $0,18 \mathrm{Abc}$ & $0,16 \mathrm{Acb}$ & 0,16 & $0,21 \mathrm{Ac}$ & $0,20 \mathrm{Abc}$ & 0,20 & $0,26 \mathrm{Ab}$ & $0,26 \mathrm{Aab}$ & 0,26 \\
\hline 10 & $2,0+$ livre & $0,07 \mathrm{Ac}$ & $0,14 \mathrm{Acb}$ & 0,10 & 0,06Ade & $0,13 \mathrm{Ac}$ & 0,09 & $0,09 \mathrm{Ab}$ & $0,24 \mathrm{Aab}$ & 0,16 \\
\hline 10 & $2,0+$ livre $^{* *}$ & $0,23 \mathrm{Ab}$ & $0,15 \mathrm{Acb}$ & 0,18 & $0,41 \mathrm{Ab}$ & $0,19 \mathrm{Bbc}$ & 0,30 & $0,19 \mathrm{Ab}$ & $0,29 \mathrm{Aab}$ & 0,24 \\
\hline 10 & $>10+12$ & $0,07 \mathrm{Ac}$ & 0,14 Acd & 0,10 & $0,15 \mathrm{Bcde}$ & $0,34 \mathrm{Aa}$ & 0,24 & $0,19 \mathrm{Bb}$ & $0,40 \mathrm{Aa}$ & 0,29 \\
\hline 10 & $1,0+$ livre & 0,09Ac & 0,09Ac & 0,09 & $0,04 \mathrm{Ae}$ & $0,13 \mathrm{Ac}$ & 0,08 & $0,11 \mathrm{Ab}$ & $0,14 \mathrm{Ab}$ & 0,12 \\
\hline 10 & $>10+6,0$ & $0,08 \mathrm{Ac}$ & $0,14 \mathrm{Acb}$ & 0,11 & 0,10 Acde & $0,21 \mathrm{Aabc}$ & 0,15 & $0,11 \mathrm{Ab}$ & $0,27 \mathrm{Aab}$ & 0,19 \\
\hline$-0,5$ & $\mathrm{AR}$ & $0,83 \mathrm{Aa}$ & $0,56 \mathrm{Ba}$ & 0,69 & $1,23 \mathrm{Aa}$ & $0,24 \mathrm{Babc}$ & 0,73 & $0,81 \mathrm{Aa}$ & $0,35 \mathrm{Bab}$ & 0,58 \\
\hline Média & & 0,21 & 0,20 & & 0,30 & 0,21 & & 0,25 & 0,30 & \\
\hline $\mathrm{CV}(\%)$ & & \multicolumn{3}{|c|}{24,18} & \multicolumn{3}{|c|}{23,36} & \multicolumn{3}{|c|}{34,43} \\
\hline Condições de & armazenamento & & 4 dias & $20^{\circ} \mathrm{C}$ & & & & $5 \operatorname{dias}$ a $20^{\circ}$ & & \\
\hline $\begin{array}{l}\text { Temperatura } \\
{ }^{\circ} \mathrm{C}\end{array}$ & $\mathrm{O}_{2}+\mathrm{CO}_{2}(\mathrm{kPa})$ & Verde-amarel & $\mathrm{Am}$ & relo & Médias & Verde & amarelo & Amarelo & & Médias \\
\hline 10 & $\mathrm{AR}^{*}$ & $0,40 \mathrm{Bb}$ & & $3 \mathrm{Aa}$ & 1,09 & & 30 & 0,43 & & $0,34 \mathrm{ab}$ \\
\hline 10 & $\mathrm{AR}^{* *}$ & $0,53 \mathrm{Ab}$ & & Acb & 0,61 & & 27 & 0,41 & & $0,33 \mathrm{ab}$ \\
\hline 10 & $2,0+$ livre & $0,29 \mathrm{Ab}$ & & Ac & 0,44 & & 14 & 0,33 & & $0,23 \mathrm{ab}$ \\
\hline 10 & $2,0+$ livre** & $0,41 \mathrm{Ab}$ & & $5 \mathrm{Ac}$ & 0,48 & & 06 & 0,44 & & $0,25 \mathrm{ab}$ \\
\hline 10 & $>10+12$ & $0,56 \mathrm{Bb}$ & & $A b$ & 0,78 & & 18 & 0,31 & & $0,24 \mathrm{ab}$ \\
\hline 10 & $1,0+$ livre & $0,27 \mathrm{Ab}$ & & Ac & 0,36 & & 08 & 0,32 & & $0,20 \mathrm{~b}$ \\
\hline 10 & $>10+6,0$ & $0,28 \mathrm{Ab}$ & & Ac & 0,43 & & 14 & 0,36 & & $0,25 \mathrm{ab}$ \\
\hline$-0,5$ & $\mathrm{AR}$ & $1,42 \mathrm{Aa}$ & & $\mathrm{Bcb}$ & 1,06 & & 51 & 0,29 & & $0,39 \mathrm{a}$ \\
\hline Média & & 0,52 & & 80 & & & $20 \mathrm{~B}$ & $0,36 \mathrm{~A}$ & & \\
\hline $\mathrm{CV}(\%)$ & & & 23, & & & & & 39,15 & & \\
\hline
\end{tabular}

*Armazenamento refrigerado; ** Aplicação de $1 \mu \mathrm{L} \mathrm{L}^{-1}$ de 1 -MCP durante 24 horas; *** Médias seguidas de mesma letra, minúscula na vertical e maiúscula na horizontal, não diferem entre si pelo teste de Duncan a $5 \%$.

etileno em todo o período, principalmente, quando colhido no ponto de maturação amarelo. Esse resultado demonstra que o armazenamento em baixa temperatura (menor que $1{ }^{\circ} \mathrm{C}$ ) com posterior exposição a $20^{\circ} \mathrm{C}$ provoca um acelerado metabolismo do fruto, acarretando um amolecimento mais rápido quando comparado com os outros tratamentos (Tabela 1).

Os frutos no ponto de colheita verdeamarelo, quando submetidos a $1,0 \mathrm{kPaO}_{2}+\mathrm{CO}_{2}$ livre, tiveram a menor produção de etileno após 1, 2, 3 e 4 dias de exposição a $20^{\circ} \mathrm{C}$, quando comparados com os dos demais tratamentos. Este resultado demonstra que a utilização de baixo $\mathrm{O}_{2}$ na temperatura de $10^{\circ} \mathrm{C}$ é mais eficiente na redução da produção de etileno quando comparada com inibidores da ação de etileno.

Após 1 e 2 dias de exposição a $20^{\circ} \mathrm{C}$, a respiração foi menor nos frutos submetidos a $1,0 \mathrm{kPa}$
$\mathrm{O}_{2}+\mathrm{CO}_{2}$ livre. Já aos 3 , 4 e 5 dias de exposição a $20^{\circ} \mathrm{C}$, a respiração foi menor nos frutos acondicionados sob $2,0 \mathrm{kPaO}_{2}+\mathrm{CO}_{2}$ livre com aplicação de $1 \mu \mathrm{L} \mathrm{L}^{-1}$ de 1 MCP.

\section{CONCLUSÕES}

$\mathrm{O}$ armazenamento à temperatura $\mathrm{de} 10^{\circ} \mathrm{C}$ com $1,0 \mathrm{kPaO}_{2}+\mathrm{CO}_{2}$ livre; $2,0 \mathrm{kPaO}_{2}+\mathrm{CO}_{2}$ livre e $2,0 \mathrm{kPaO}$ $+\mathrm{CO}_{2}$ livre com aplicação de $1 \mu \mathrm{L} \mathrm{L}^{-1}$ de $1-\mathrm{MCP}$ proporciona maior porcentagem de frutos firmes e menor incidência de podridão e escurecimento da casca em caquis "Fuyu", após 17 dias de armazenamento e durante a exposição dos frutos a $20^{\circ} \mathrm{C}$. A aplicação de 1-MCP não é eficiente na manutenção da consistência firme no armazenamento refrigerado na temperatura de 
Tabela 5 - Respiração em caquis cv. "Fuyu” após 17 dias de armazenamento e durante a exposição a $20^{\circ} \mathrm{C}$. Santa Maria, RS, 2005.

\begin{tabular}{|c|c|c|c|c|c|c|c|c|c|c|}
\hline \multirow{2}{*}{\multicolumn{2}{|c|}{ Condições de armazenamento }} & \multicolumn{9}{|c|}{ Respiração $\left(\mathrm{mLCO}_{2} \mathrm{~kg}^{-1} \mathrm{~h}^{-1}\right)$} \\
\hline & & \multicolumn{3}{|c|}{1 dia a $20^{\circ} \mathrm{C}$} & \multicolumn{3}{|c|}{2 dias a $20^{\circ} \mathrm{C}$} & \multicolumn{3}{|c|}{3 dias a $20^{\circ} \mathrm{C}$} \\
\hline $\begin{array}{l}\text { Temperatura } \\
{ }^{\circ} \mathrm{C}\end{array}$ & $\mathrm{O}_{2}+\mathrm{CO}_{2}(\mathrm{kPa})$ & $\begin{array}{l}\text { Verde- } \\
\text { amarelo }\end{array}$ & $\mathrm{Am}$ & Médias & $\begin{array}{c}\text { Verde } \\
\text { amarelo }\end{array}$ & Amarelo & Médias & $\begin{array}{l}\text { Verde- } \\
\text { amarelo }\end{array}$ & Amarelo & Médias \\
\hline 10 & $\mathrm{AR}^{*}$ & 15,12 & & $16,73 b^{* * *}$ & 9,78 & 9,86 & $9,82 \mathrm{a}$ & 10,59 & 12,31 & $11,45 \mathrm{a}$ \\
\hline 10 & $\mathrm{AR}^{* *}$ & 13,60 & & $14,37 \mathrm{c}$ & 7,85 & 8,13 & $7,98 \mathrm{~b}$ & 8,84 & 10,45 & $9,64 b$ \\
\hline 10 & $2,0+$ livre & 8,88 & & $9,80 \mathrm{e}$ & 6,11 & 7,08 & $6,59 \mathrm{~cd}$ & 7,01 & 9,36 & $8,19 \mathrm{c}$ \\
\hline 10 & $2,0+$ livre** & 8,46 & & $9,40 \mathrm{e}$ & 5,30 & 6,64 & $5,97 \mathrm{~d}$ & 5,46 & 6,59 & $6,02 d$ \\
\hline 10 & $>10+12$ & 12,02 & & $12,56 \mathrm{~cd}$ & 6,96 & 8,47 & $7,71 b c$ & 9,45 & 9,76 & $9,60 \mathrm{~b}$ \\
\hline 10 & $1,0+$ livre & 8,51 & & $9,30 \mathrm{e}$ & 6,59 & 7,02 & $6,81 \mathrm{~cd}$ & 7,46 & 7,94 & $7,69 \mathrm{c}$ \\
\hline 10 & $>10+6,0$ & 10,01 & & $11,58 \mathrm{de}$ & 6,41 & 7,27 & $6,84 \mathrm{~cd}$ & 7,67 & 9,82 & $8,74 b c$ \\
\hline$-0,5$ & $\mathrm{AR}$ & 19,06 & & $20,06 a$ & 9,87 & 10,05 & $9,96 \mathrm{a}$ & 12,28 & 12,73 & $12,50 \mathrm{a}$ \\
\hline Média & & $11,96 \mathrm{~B}$ & 13,9 & & $7,36 \mathrm{~B}$ & $8,06 \mathrm{~A}$ & & $8,59 \mathrm{~B}$ & $9,87 \mathrm{~A}$ & \\
\hline $\mathrm{CV}(\%)$ & & 12,10 & & & & 9,10 & & & 9,63 & \\
\hline \multicolumn{2}{|c|}{ Condições de armazenamento } & \multicolumn{4}{|c|}{4 dia a $20^{\circ} \mathrm{C}$} & \multicolumn{5}{|c|}{5 dia a $20^{\circ} \mathrm{C}$} \\
\hline $\begin{array}{c}\text { Temperatura } \\
{ }^{\circ} \mathrm{C}\end{array}$ & $\mathrm{O}_{2}+\mathrm{CO}_{2}(\mathrm{kPa})$ & Verde-an & arelo & Amarelo & Médias & \multicolumn{2}{|c|}{ Verde-amarelo } & \multicolumn{2}{|c|}{ Amarelo } & Médias \\
\hline 10 & $\mathrm{AR}^{*}$ & \multicolumn{2}{|c|}{$20,98 \mathrm{Aa}$} & $17,98 \mathrm{Aa}$ & 19,48 & \multicolumn{2}{|r|}{10,21} & \multicolumn{2}{|c|}{10,97} & $10,59 a$ \\
\hline 10 & $\mathrm{AR}^{* *}$ & \multicolumn{2}{|c|}{$13,19 \mathrm{Bbc}$} & $21,53 \mathrm{Aa}$ & 17,36 & \multicolumn{2}{|r|}{7,87} & \multicolumn{2}{|c|}{8,70} & $8,28 \mathrm{abc}$ \\
\hline 10 & $2,0+$ livre & \multicolumn{2}{|c|}{ 7,12Ade } & $9,66 \mathrm{Ab}$ & 8,39 & \multicolumn{2}{|r|}{7,70} & \multicolumn{2}{|c|}{10,70} & $9,19 \mathrm{a}$ \\
\hline 10 & $2,0+$ livre $^{* *}$ & \multicolumn{2}{|c|}{$5,85 \mathrm{Ae}$} & $6,47 \mathrm{Ab}$ & 6,16 & \multicolumn{2}{|r|}{5,03} & \multicolumn{2}{|c|}{7,08} & $6,05 \mathrm{c}$ \\
\hline 10 & $>10+12$ & \multicolumn{2}{|c|}{$11,52 \mathrm{Abcd}$} & $11,20 \mathrm{Ab}$ & 11,36 & \multicolumn{2}{|r|}{9,25} & \multicolumn{2}{|c|}{9,99} & $9,62 \mathrm{a}$ \\
\hline 10 & $1,0+$ livre & \multicolumn{2}{|c|}{ 8,32Acde } & $9,01 \mathrm{Ab}$ & 8,66 & & 6,70 & 6,52 & & $6,60 \mathrm{bc}$ \\
\hline 10 & $>10+6,0$ & 10,49 & cde & $9,68 \mathrm{Ab}$ & 10,08 & & 7,70 & 10,0 & & $8,86 \mathrm{ab}$ \\
\hline$-0,5$ & $\mathrm{AR}$ & 15,99 & & $11,42 \mathrm{Ab}$ & 13,70 & & 9,54 & 11,3 & & $10,44 a$ \\
\hline Média & & 11,68 & & 12,12 & & & 7,99B & 9,41 & & \\
\hline $\mathrm{CV}(\%)$ & & & & 19,01 & & & & 17,4 & & \\
\hline
\end{tabular}

*Armazenamento refrigerado; ** Aplicação de $1 \mu \mathrm{L} \mathrm{L}^{-1}$ de 1-MCP durante 24 horas; *** Médias seguidas de mesma letra, minúscula na vertical e maiúscula na horizontal, não diferem entre si pelo teste de Duncan a $5 \%$.

$10^{\circ} \mathrm{C}$. No entanto, a aplicação de 1-MCP sob atmosfera controlada é mais eficiente no ponto de colheita verdeamarelo.

\section{REFERÊNCIAS}

BEN-ARIE, R. et al. Extending the shelf life of 'Triumph' persimmon after storage with 1-MCP. Alon Hanotea, v.55, n.12, p.524-527, 2001.

BLANKENSHIP, S. Potential MCP revolution. Acessado em 07 dez. de 2001. On line. Disponível na internet http:// postharvest.tfrec. wsu.edu/pgDisplay.php?article $=$ PC2000E.

BRACKMANN, A.; CHITARRA, A.B. Atmosfera controlada e atmosfera modificada. In: BOREM, F.M. Armazenamento e processamento de produtos agrícolas. Lavras: UFLA/ SBEA, 1998. 282p. p.133-170.
BRACKMANN, A. et al. Frigoconservação de caquis (Diospyrus kaki, L.) das cultivares Fuyu e Rama Forte. Ciência Rural, v.27, n.4, p.561-565, 1997.

BRACKMANN, A.; SAQUET, A.A. Efeito da temperatura e condições de atmosfera controlada sobre a conservação de caqui (Diospyros kaki, L.). Ciência Rural, v.25, n.2, p.215-218, 1995.

CRISOSTO, C.H. et al. Persimmons: recomendations for mantaining postharvest quality. Acessado em 15 de out. de 2004. On line. Disponivel na internet http// postharvest.ucdavis.edu/produce/storage.htlm.

DA SILVA, R. et al. Pectinases, hemicelulases e celulases, ação, produção e aplicação no processamento de alimentos: revisão. Boletim da Sociedade Brasileira de Ciência e Tecnologia dos Alimentos, v.31, n.2, p.249-260, 1997.

DONAZZOLO, J.; BRACKMANN, A. Efeito do $\mathrm{CO}_{2}$ em atmosfera controlada na qualidade de caqui (Diospyros kaki, 
L.) cv. "Fuyu". Revista Brasileira de Agrociência, v.8, n.3, p.241-245, 2002.

DONG, J.G. et al. Purification and characterization of 1aminocyclopropane-1-carboxylate oxidase from apple fruit. Proceedings of the National Academy of Science of USA, v.89, p.9789-9793, 1992.

GORINI, F.L.; TESTONI, A. Raccolta e conservazione e trasformazione dei frutti de kaki. Annali dell' Istituto Sperimentale per la Valorizzazione Tecnologica dei Prodotti Agricoli, v.19, p.249-258, 1988.

HARIMA, S. et al. Extending shelf-life of astringent persimmon (Diospyrus kaki, L.) fruits dy 1-MCP. Postharvest Biology and Technology, v.29, n.1, p.318-323, 2003.

LEE, S.K. et al. Factors involved skin browning of nonastringents 'Fuyu' persimmon. Acta Horticulturae, n.343, p.300-303, 1993.

LEE, Y.J. Browning disorders of "Fuyu" persimmon fruit caused by low oxygen and low temperature in modified atmosphere storage. Journal of the Korean Society for Horticultural Science, v.42, n.6, p.725-731, 2001.

LEE, Y.M. et al. Effects of oxygen and carbon dioxide concentration in PE film bag on blackening and flesh browning during MA storage of 'Fuyu' persimmon fruit. Journal of the Korean Society for Horticultural Science, v.40, n.5, p.585$590,1999$.

LEE, Y.J. et al. Effects of low oxygen and high carbon dioxide concentrations on modified atmosphere-related disorder of 'Fuyu' persimmon fruit. Acta Horticulturae, n.601, p.171176, 2003.

MULLINS, E.D. et al. Consequences on ethylene metabolism of inactivating the ethylene receptor sites in discase nonclimateric fruit. Postharvest Biology and Techenology, n.2, v.19, p.155-164, 2000.
NEUWALD, D.A. Armazenamento de caqui (Diospyros kaki L.) cultivar Fuyu em atmosfera controlada. 2004. 66f. Dissertação (Mestrado em Agronomia) - Universidade Federal de Santa Maria, Santa Maria.

PARK, Y.S. Changes in fruit skin blackening, phenolic acids and ethanol production of non-astringent 'Fuyu' persimmon fruits during CA storage. In: INTERNATIONAL CONTROLLED ATMOSPHERE RESEARCH CONFERENCE, 7., 1997, Davis. Proceedings... Davis: University of California, 1997. p.170-176.

PRUSKY, D. et al. Effect of modified atmosphere for control of black spot, caused by Alternaria alternate, on stored persimmon fruits. Phytopathology, v.87, n.2, p.203-208, 1997.

SARGENT, S.A. et al. Storage characteristics of 'Fuyu' persimmons. Proceedings of the Florida State Horticultural Society, v.106, p.131-134, 1993.

SISLER, E.C.; SEREK, M. Compounds controlling the ethylene receptor. Acesso em 04 dez. 2004. On line. Disponível em: http://ejournal.sinica.edu.tw/bbas/content/1999/ 1/bot 41-01.pdf $>$.

TAIZ, L.; ZEIGER, E. Plant physiology. 2.ed. Redwood City: Benjamin/Cummings, 1998. 565p.

VIDRIH, R. et al. Storing of persimmon fruit under controlled atmosphere conditions. In: INTERNATIONAL HORTICULTURAL CONGRESS, 23., 1990, Firenze. Abstracts... Firenze: ISHS, 1990. V.2, p.3312.

WOOLF, A. B. et al. Reduction of chilling injury in the sweet persimmons 'Fuyu' during storage by dry air heat treatments. Postharvest Biology and Technology, Amsterdam, v.11, n.3, p.155-164, 1997. 